\title{
A avaliação do sistema de referência e contrarreferência na atenção secundária em Odontologia
}

\author{
Assessment of the reference and counter-reference system in \\ secondary dentistry care
}

\author{
Gabriela Novo Borghi" \\ Fabiana de Lima Vazquez** \\ Karine Laura Cortellazzi*** \\ Luciana Miranda Guerra*** \\ Jaqueline Vilela Bulgareli**** \\ Antonio Carlos Pereira
}

\section{Resumo}

Para o alcance da integralidade em saúde bucal, é necessária a organização da "porta de entrada" desse sistema, ou seja, da atenção básica e, sobretudo, da sua interligação com a atenção secundária. Objetivo: avaliar o sistema de referência e contrarreferência nos procedimentos de atenção secundária na área odontológica em relação ao fluxo, à qualidade de atendimento e ao acesso. Materiais e método: trata-se de um estudo do tipo observacional exploratório para análise de fluxo, qualidade de atendimento e acesso dos encaminhamentos para a atenção secundária de 12 Unidades de Saúde da Família (USF) e 20 Unidades Básicas de Saúde (UBS) com equipes de saúde bucal. Na primeira fase do estudo, foram identificados os usuários $(n=101)$ que procuraram o Centro de Especialidades Odontológicas (CEO) para tratamento no período de agosto a dezembro de 2011. Realizou-se entrevista estruturada com questões abertas referentes ao tempo de espera para $o$ atendimento especializado desde o encaminhamento pela atenção básica, qualidade do atendimento, satisfação do usuário e acesso. Na segunda fase do estudo, foram aplicadas para os mesmos pacientes mais duas questões referentes ao retorno nas consultas da atenção básica. Resultados: a maioria dos entrevistados relatou ter esperado de uma semana a um mês para o atendimento na atenção especializada, 95\% deles não tiveram queixas do atendimento e $66,66 \%$ dos pacientes atendidos no CEO não retornaram para a atenção básica. Conclusão: nota-se a existência de um fluxo de atendimento de usuários da atenção básica para a atenção secundária em saúde bucal em Piracicaba $(S P)$, tendo os usuários demonstrado satisfação com o atendimento. Contudo, a contrarreferência foi significativamente deficiente, considerando que a maioria dos pacientes não voltou para a atenção básica.

Palavras-chave: Saúde bucal. Acesso aos serviços de saúde. Saúde pública. Equidade.

Graduanda da Faculdade de Odontologia de Piracicaba, FOP/UNICAMP, Departamento de Odontologia Social, Piracicaba, SP, Brasil.

Doutoranda em Saúde Coletiva da Faculdade de Odontologia de Piracicaba, FOP/UNICAMP, Departamento de Odontologia Social, Piracicaba, SP, Brasil. Pós-doutoranda em Saúde Coletiva da Faculdade de Odontologia de Piracicaba, FOP/UNICAMP, Departamento de Odontologia Social, Piracicaba, SP, Brasil. Pós-doutoranda em Saúde Coletiva da Faculdade de Odontologia de Piracicaba, FOP/UNICAMP, Departamento de Odontologia Social, Piracicaba, SP, Brasil. Doutoranda em Saúde Coletiva da Faculdade de Odontologia de Piracicaba, FOP/UNICAMP, Departamento de Odontologia Social, Piracicaba, SP, Brasil.

Professor doutor da Faculdade de Odontologia de Piracicaba, FOP/UNICAMP, Departamento de Odontologia Social, Piracicaba, SP, Brasil. 


\section{Introdução}

Considerando o princípio da integralidade e a construção das redes de atenção no Sistema Único de Saúde (SUS), a atenção secundária pode refletir a resolutividade da atenção básica, apoiando a organização das ações e dos serviços de saúde bucal no âmbito da média complexidade ${ }^{1}$.

A integralidade em saúde bucal, além de princípio do SUS, é direito constitucional de todo cidadão $0^{2}$, e, para o seu alcance, é necessária a organização da "porta de entrada" desse sistema, ou seja, da atenção básica e, sobretudo, da sua interligação com a atenção secundária.

Nas últimas décadas, evidenciou-se a ênfase na promoção da saúde e no fortalecimento da Atenção Básica em Saúde Bucal, por meio de ações realizadas nas Unidades de Saúde da Família (USF) e nas Unidades Básicas de Saúde (UBS).

Morris e Burke ${ }^{3}$ (2001) definiram quatro características importantes para a interligação entre a atenção básica e a secundária em odontologia: 1) acesso indiscriminado e sem barreiras à atenção especializada após encaminhamento; 2) sistema de referência, por meio do qual todo serviço não disponível na atenção básica seja ofertado na atenção especializada; 3) encaminhamento eficiente e adequado com contrarreferência para a atenção primária ao término do tratamento especializado; 4) retorno facilitado à atenção secundária sempre que necessário.

A maior parte da assistência odontológica no SUS tem-se restringido quase que somente à atenção básica e com grande demanda reprimida, refletindo nas desigualdades sociais do país a utilização dos serviços odontológicos ${ }^{4}$.

Segundo o Ministério da Saúde (MS), no ano 2000 , a atenção secundária apresentava baixos percentuais (apenas 3,3\%) dentro do escopo de procedimentos odontológicos ${ }^{5}$. Figueiredo e Góes, em 2009, afirmaram haver insuficiência de serviços especializados, o que compromete a eficiência da referência e contrarreferência em saúde bucal ${ }^{6}$. Esses fatos culminaram com a preocupação do governo federal em incentivar a criação dos Centros de Especialidades Odontológicas (CEO) dentro da Política Nacional de Saúde Bucal (PNSB). Nota-se o crescente avanço na implantação dos CEO em todo o país, os quais passaram de 100 para 832 no período de 2004 a 2010 , com aumento de $732 \%$.

A construção da integralidade em saúde bucal requer a valorização da atenção secundária com o fortalecimento e desenvolvimento do sistema de referência e contrarreferência, e as dificuldades apresentadas nesse sentido são, definitivamente, seus entraves. Superar, ou, ao menos, amenizar esses entraves pode abreviar a distância entre o usuário e a resolução de suas necessidades em saúde bucal ${ }^{8}$.
Contudo, para que a resolubilidade prevista seja alcançada, a atenção secundária terá que assegurar aos usuários o acesso a consultas, procedimentos e exames especializados ${ }^{7}$. A principal estratégia de operacionalização adotada para a atenção especializada em saúde bucal foi a implantação dos CEO, que são estabelecimentos de saúde classificados no Cadastro Nacional de Estabelecimentos de Saúde (CNES) como Clínica Especializada ou Ambulatório de Especialidade. Tais serviços são, entretanto, apenas uma das vertentes assistenciais da atual PNSB' 9 .

O CEO tem como atribuição oferecer à população, minimamente, os serviços de: diagnóstico bucal, com ênfase no diagnóstico e na detecção do câncer de boca; periodontia especializada; cirurgia oral menor dos tecidos moles e duros; endodontia; prótese e atendimento a portadores de necessidades especiais. Esses centros de especialidades constituem uma das frentes de atuação do Programa Brasil Sorridente. O tratamento oferecido é uma continuidade do trabalho realizado pela rede de atenção básica, e, no caso dos municípios que estão na Estratégia de Saúde da Família, pelas respectivas equipes de saúde bucal. O fluxo da atenção consiste em que os profissionais da atenção básica sejam responsáveis pelo primeiro atendimento ao paciente e pelo encaminhamento dos casos mais complexos aos $\mathrm{CEO}^{1}$.

Os CEO podem ser classificados em: CEO tipo I - com três cadeiras odontológicas; CEO tipo II, com quatro ou mais cadeiras e CEO tipo III, com no mínimo sete cadeiras ${ }^{1}$.

O papel complementar dos diferentes níveis de atenção à saúde remete-nos ao conceito da integralidade, a qual exige que os serviços sejam organizados de forma a garantir ao indivíduo e à coletividade a promoção, a proteção e a recuperação da saúde, de acordo com as necessidades de cada um em todos os níveis de complexidade do sistema ${ }^{10}$.

O objetivo do trabalho foi avaliar o sistema de referência e contrarreferência nos procedimentos de atenção secundária na área odontológica em relação ao fluxo, à qualidade de atendimento e ao acesso.

\section{Materiais e método}

Este trabalho foi submetido à aprovação do Comitê de Ética em Pesquisa (CEP) da FOP - UNICAMP, conforme resolução 196/96, de 10/10/1996 do Conselho Nacional de Saúde do MS. Os procedimentos somente foram iniciados após terem sido devidamente autorizados pelo CEP e pelas autoridades envolvidas no estudo.

Trata-se de um estudo do tipo observacional exploratório para análise de fluxo, qualidade de atendimento e acesso dos encaminhamentos para a atenção secundária de 12 USF e 20 UBS que possuem dentista. 


\section{Descrição do universo e amostra}

Atualmente, Piracicaba ocupa uma área de $795,70 \mathrm{Km}^{2}$ e conta com uma população de aproximadamente 364.571 habitantes (segundo dados do IBGE/2011), distribuída em cinco regiões de saúde: Norte, Sul, Leste, Oeste e Centro.

A rede pública de saúde em Piracicaba é composta por 57 Unidades de Saúde, sendo 34 USF e 23 UBS, um Centro de Especialidades Médicas, dois CEO, quatro Unidades de Pronto-Atendimento Médico (UPA) e uma Unidade de Pronto-Atendimento Odontológico (Serviço de Urgências Bucais - SUB), uma policlínica e dois hospitais de referência.

O serviço odontológico no município está inserido em 12 USF módulo I (cirurgião-dentista e auxiliar de saúde bucal). Existem 20 UBS com dentista, seis delas contavam com auxiliar, cinco unidades incluíram esse profissional na equipe a partir do final de 2009 e as demais trabalham apenas com o cirurgião-dentista. Os CEO atendem as especialidades de endodontia, periodontia, cirurgia bucomaxilo, odontopediatria, dentística, pacientes especiais e bebês de 0 a 4 anos, anexo ao CEO. Foi realizado um sorteio para a seleção do CEO no qual foi feita a coleta dos dados.

\section{Critérios de inclusão/exclusão}

Os critérios de inclusão foram pacientes maiores de 12 anos, com ausência de dificuldades de comunicação ou neuromotoras, sem restrição de gênero e idade, moradores de bairros atendidos pelas USF e UBS e que foram referenciados para atendimento no CEO. A participação deu-se após assinatura do termo de consentimento livre e esclarecido. Indivíduos que não concordaram em participar do estudo e os ausentes no dia do exame foram excluídos.

\section{Coleta de dados}

Inicialmente, foram identificados todos os usuários que procuraram o CEO para tratamento na atenção secundária. A seguir, foi realizada uma entrevista estruturada com questões abertas referentes ao tratamento realizado, seguindo o roteiro:

$1^{\mathrm{a}}$ fase: foram aplicadas questões para os usuários referenciados ao CEO, no momento em que o paciente aguardava atendimento na sala de espera.

1- Quanto tempo esperou para ter atendimento especializado - desde o encaminhamento até a primeira consulta?

2- Como foi ou está sendo seu atendimento?

3- Você possui alguma queixa com relação ao atendimento?

4- Você teve alguma dificuldade com relação ao atendimento?
Os dados foram coletados semanalmente, no período de $1^{\circ}$ de agosto de 2011 a 31 de dezembro de 2011, junto ao CEO sorteado, totalizando 101 indivíduos.

$2^{\mathrm{a}}$ fase: após quatro meses da primeira entrevista, os mesmos usuários foram contatados e foram feitas as seguintes perguntas:

1- Você voltou a ver seu dentista após o tratamento especializado?

2 - Se não procurou o $\mathrm{CD}$, por quê?

\section{Análise estatística}

O estudo foi de natureza quantitativa, e a análise estatística foi descritiva.

\section{Resultados}

Na primeira fase do trabalho, foram entrevistados 101 pacientes referenciados das UBS ou USF para atenção especializada no Centro de Especialidades Odontológicas.

A Tabela 1 mostra que, quanto ao tempo de espera para o atendimento especializado após o encaminhamento pela atenção básica, $15,84 \%$ esperaram uma semana entre o encaminhamento e a primeira consulta no CEO II; 28,71\% aguardaram 15 dias; $35,64 \%$, um mês e $17,82 \%$ precisaram aguardar mais de um mês.

Tabela 1 - Frequência de respostas em relação ao tempo de espera para atendimento desde o encaminhamento até a primeira consulta

\begin{tabular}{l|c}
\hline \multicolumn{1}{|c|}{ Tempo de espera } & $\%$ \\
\hline 1 semana & $15,84 \%$ \\
15 dias & $28,71 \%$ \\
1 mês & $35,64 \%$ \\
Mais de 1 mês & $17,82 \%$ \\
3 meses & $1,99 \%$ \\
\hline
\end{tabular}

Em relação à qualidade do atendimento no CEO, 30,69\% o classificaram como ótimo; a maioria, $68,31 \%$, o considerou bom e apenas $0,99 \%$ relataram que 0 atendimento foi regular, conforme Tabela 2.

Tabela 2 - Frequência de respostas sobre a qualidade do atendimento

\begin{tabular}{l|c}
\multicolumn{1}{c|}{ Qualidade do atendimento } & $\%$ \\
\hline Ótimo & $30,69 \%$ \\
Bom & $68,32 \%$ \\
Regular & $0,99 \%$ \\
\hline
\end{tabular}

Em relação à questão 1.3 - Você possui alguma queixa com relação ao atendimento? -, a maioria dos entrevistados, $95,05 \%$, respondeu negativamente, e apenas $4,95 \%$ relatou ter alguma queixa (Tabela 3 ). 
Tabela 3 - Frequência de respostas em relação à existência ou não de queixas sobre $o$ atendimento

\begin{tabular}{|c|c|}
\hline Existência de queixa & $\%$ \\
\hline Sim & $4,95 \%$ \\
\hline Não & $95,05 \%$ \\
\hline
\end{tabular}

À questão 1.4 - Você possui alguma dificuldade com relação ao tratamento? -, 83,16\% responderam que não, $0,99 \%$ apontaram dificuldade para marcar horário, 7,92\% consideraram o local mal sinalizado, difícil de ser encontrado, também por falta de informação do endereço correto; por fim, 7,92\% o consideraram muito distante de sua casa (Tabela 4).

Tabela 4- Frequência de respostas em relação à existência ou não de dificuldade para realizar o tratamento

\begin{tabular}{l|c}
\hline \multicolumn{1}{c|}{ Dificuldade } & $\%$ \\
\hline Nenhuma dificuldade & $83,16 \%$ \\
$\begin{array}{l}\text { Dificuldade para marcar horário } \\
\text { Consideraram o local mal sinalizado, }\end{array}$ & $0,99 \%$ \\
$\begin{array}{l}\text { sem informações e difícil de ser } \\
\text { encontrado }\end{array}$ & $7,92 \%$ \\
$\begin{array}{l}\text { Consideraram o local distante de sua } \\
\text { casa }\end{array}$ & $7,92 \%$ \\
\hline
\end{tabular}

$\mathrm{Na}$ segunda fase do projeto, foram aplicadas, para os mesmos pacientes, mais duas questões por meio de contato telefônico.

A Tabela 5 demonstra esses resultados. À questão 2.1 - Você voltou a ver seu dentista após o tratamento especializado? -, 62 usuários, 61,38\%, responderam que SIM, que voltaram para o atendimento básico em suas unidades, e 39 usuários, $38,61 \%$, apontaram que não voltaram às unidades para dar continuidade ao tratamento.

Tabela 5 - Distribuição e frequência de respostas dos pacientes que fizeram o tratamento especializado e que voltaram ou não para a atenção básica

\begin{tabular}{c|c|c}
\hline Atenção básica & $\%$ & $\%$ \\
\hline Sim - Voltaram para o atendimento básico & 61,38 & 62 \\
Não - Não voltaram para o atendimento básico & 38,61 & 39 \\
\hline
\end{tabular}

A Tabela 6 mostra os resultados da questão 2.2. Vinte e seis dos 39 entrevistados, ou seja, $66,66 \%$ dos que não retornaram para a atenção básica responderam apenas que não procuraram o $\mathrm{CD}$ nas unidades, sem fornecer outras informações; no entanto, 9 dos 39 pacientes, ou seja, 23,07\%, não procuraram o $\mathrm{CD}$ nas unidades porque entenderam não precisar mais de atendimento; por fim, 4 usuários, $10,25 \%$, não retornaram porque disseram não ter conseguido marcar a consulta.
Tabela 6 - Distribuição e frequência das respostas dos entrevistados para não retornarem ao atendimento na atenção básica

\begin{tabular}{l|c}
\hline Motivos para não retornar à atenção básica & $\begin{array}{c}\text { № de } \\
\text { usuários }\end{array}$ \\
\hline $\begin{array}{l}\text { Apenas não voltaram } \\
\text { Não voltaram, entenderam não precisar mais } \\
\text { de atendimento }\end{array}$ & 9 \\
$\begin{array}{l}\text { Não voltaram, pois não conseguiram marcar } \\
\text { consulta }\end{array}$ & 4 \\
\hline
\end{tabular}

Em relação ao gênero, $70 \%$ dos usuários eram do sexo feminino e $30 \%$ do sexo masculino (Tabela 7).

\begin{tabular}{l|c}
\multicolumn{2}{|c}{ Tabela 7 - Caracterização dos usuários por gênero } \\
\hline \multicolumn{1}{|c|}{ Gênero } & $\mathrm{n}$ \\
\hline Feminino & 70 \\
Masculino & 31 \\
\hline
\end{tabular}

\section{Discussão}

Este estudo identificou importantes achados em relação ao acesso, ao fluxo e à qualidade do atendimento dos usuários referenciados para atenção secundária em saúde bucal no município de Piracicaba, SP.

Quanto ao gênero, comprovou-se que a maioria dos usuários que procuraram o serviço de atenção secundária correspondeu a mulheres, fato este, provavelmente, explicado pelo horário de funcionamento do serviço, que não é estendido além do comercial, refletindo na dificuldade de acesso ao serviço por parte daqueles que trabalham, principalmente, homens trabalhadores. Além disso, o resultado corrobora o estudo de Gomes et al. ${ }^{11}$, que encontraram menor frequência de homens utilizando os serviços de saúde.

O tempo de espera foi consideravelmente reduzido, tendo a maioria relatado de uma semana a um mês. Tal resultado foi ao encontro da pesquisa de Vazquez et al. (2011), que apurou, em duas regiões de saúde do município de Campinas (SP), espera de 30 dias para a maioria dos pacientes referenciados para a atenção secundária em saúde bucal ${ }^{8}$.

As respostas dos pacientes sobre qualidade do atendimento, classificando-o, na maioria das vezes, como bom ou ótimo, revelam que há resolutividade e acolhimento adequado. Isso foi confirmado quando cerca de $95 \%$ dos entrevistados relataram não ter queixas do atendimento.

Em relação ao acesso dos pacientes aos procedimentos especializados, embora a maioria tenha relatado não ter encontrado dificuldade, há que se considerar que cerca de $15 \%$ dos referenciados apontaram entraves passíveis de solução mediante ações administrativas, de gestão e de de comunica- 
ção entre as unidades e os usuários, como: melhor sinalização da unidade de referência, descentralização dos serviços secundários para bairros mais distantes e melhor informação dos usuários, pelas unidades básicas, sobre a localização da unidade de referência. A execução de tais ações, certamente, implicará em diminuição de faltas, o que, por sua vez, impactará na otimização de recursos.

Dessa forma, é importante reforçar a afirmação da Organização Pan-Americana de Saúde (OPAS): "0 conceito de oferta de serviços, implícito na definição de cobertura, significa que tais serviços sejam acessíveis aos membros da comunidade e, desse modo, satisfaçam às suas necessidades no tocante à saúde"12.

Assim, a mera existência ou disponibilidade de um serviço não garante a acessibilidade. É necessário subdividir esse conceito em acessibilidade geográfica (a distância, o tempo de locomoção e os meios de transporte devem determinar a localização dos estabelecimentos, e não áreas teóricas de jurisdição), acessibilidade financeira (os pagamentos ou contribuição para utilização dos serviços não devem constituir obstáculos), acessibilidade cultural (não deve haver conflito entre os padrões técnicos e administrativos dos serviços e os hábitos, padrões culturais e costumes das comunidades em que sejam prestados) e acessibilidade funcional (os serviços devem ser prestados oportunamente e em caráter contínuo, bem como estar disponíveis a qualquer momento, atender à demanda real e incluir um sistema de referência que assegure fácil acesso ao nível de assistência que se requer) ${ }^{12}$.

Embora a maioria dos pacientes atendidos tenha retornado para a unidade básica de origem, há um importante contingente $(38,61 \%)$ que não retornou. Isso também é um entrave à integralidade, na medida em que não há continuidade da atenção. A compreensão desse fato ficou prejudicada no presente trabalho, uma vez que a maioria dos entrevistados restringiu-se a informar que "não voltou", não fornecendo, assim, detalhes que poderiam esclarecer melhor as dificuldades. Contudo, daqueles que especificaram os motivos, infere-se que há necessidade de que o profissional especialista empenhe-se melhor na contrarreferência e, sobretudo, na informação dos pacientes sobre a importância desse retorno na resolutividade final do caso.

Diante disso, Rocha e Bercht ${ }^{13}$ (2000) analisaram o abandono do tratamento em um Centro de Saúde em Porto Alegre/RS, onde os resultados revelaram como principais causas a impossibilidade de faltar ao trabalho, o tempo de duração do tratamento demasiado longo e as causas externas ao serviço, como doenças, gravidez e mudança de domicílio, o que pode explicar, também, o percentual encontrado no presente estudo.

Ressalta-se que o impacto das ações de saúde bucal na atenção básica tem evidente progresso do ponto de vista da universalidade, equidade e integralidade. Esta análise contribui na avaliação da organização da atenção básica, considerando que a integralidade da atenção efetiva-se na coordenação do cuidado pela Atenção Primária em Saúde (APS) integrada à rede nos demais níveis de atenção (neste estudo, atenção secundária), que caminha desde o acesso ao acompanhamento até a resolução da necessidade de saúde existente.

No entanto, percebe-se, neste estudo, que há necessidade de se empenhar esforços na organização da demanda da atenção básica, a fim de se garantir o acesso desses pacientes que retornam da atenção secundária, contribuindo para o acompanhamento do cuidado em saúde dos usuários que perpassam pela rede de atenção à saúde bucal do município.

\section{Conclusão}

A existência de um fluxo de atendimento de usuários da atenção básica para a atenção secundária em saúde bucal em Piracicaba demonstra estar em construção e ter boa aceitação por parte de seus usuários. Carece, contudo, de ajustes, principalmente do ponto de vista de gestão das unidades e de sua comunicação com os pacientes.

É fundamental a reflexão sobre a organização da contrarreferência dos pacientes para a atenção básica, no sentido de se garantir a integralidade da atenção à saúde bucal, fator primordial para assegurar a longitudinalidade do cuidado e a qualidade do atendimento.

\section{Abstract}

Achieving integrality in oral health requires the organization of this system's "gateway", that is, of primary care and foremost of its connection to secondary care. Objective: to assess the reference and counter-reference system of secondary care procedures in dentistry concerning flow, service quality, and access. Materials and method: observational exploratory study of flow analysis, service quality, and access of referrals to secondary care of 12 Family Health Units (FHU) and 20 Basic Health Units (BHU) with oral health teams. In the first stage of the study users $(n=101)$ who sought the Dental Specialty Center (DSC) for treatment from August to December 2011 were identified. We conducted structured interviews with open questions about the waiting time for specialized care from the time of referral provided by primary care, service quality, user satisfaction, and access. In the second stage of the study we addressed the same patients with two more questions regarding their return for primary care appointments. Results: most respondents reported having waited from 1 week to 1 month for care in the specialized center, 95\% of them had no complaints, and $66.66 \%$ of the patients treated at the DSC did not return for primary care. Conclusion: there is a service flow from users of primary care to secondary care in the oral health system in the 
city of Piracicaba (SP) - Brazil. Users were satisfied with the service; however, counter-reference was significantly deficient since most patients did not return to primary care.

Keywords: Oral health. Health services accessibility. Public health. Equity.

\section{Referências}

1. Brasil. Ministério da Saúde. Portaria no 599 GM, de 23 de março de 2006. Brasília: Ministério da Saúde; 2006.

2. Brasil. Lei no 8080, de 19 de setembro de 1990. Diário Oficial da União, Brasília, 1990, seção 20, p. 18055-9.

3. Morris AJ, Burke FJT. Primary and secondary dental care: how ideal is the interface? British Dental J 2001; 191(12):666-70.

4. Matos DL, Lima-Costa MFF, Guerra HL, Marcenes W. Projeto Bambuí: estudo de base populacional dos fatores associados com o uso regular dos serviços odontológicos em adultos. Cad Saúde Pública 2001; 17:661-8.

5. Brasil. Ministério da Saúde. Secretaria de Vigilância em Saúde. Departamento de Análise de Situação de Saúde. Saúde Brasil 2008: 20 anos de Sistema Único de Saúde (SUS) no Brasil. Brasília: Ministério da Saúde; 2009.

6. Figueiredo N, Goes PSA. Construção da atenção secundária em saúde bucal: um estudo sobre os Centros de Especialidades Odontológicas em Pernambuco, Brasil, Faculdade de Odontologia, Universidade de Pernambuco, Camaragibe, Brasil. Cad Saúde Pública 2009; 25 (2):259-67.

7. Brasil. Portal da Saúde. Brasil Sorridente [acesso 2013 Set 02]. Disponível em URL: http://portal.saude.gov.br/saude/ visualizar_texto.cfm?idtxt $=19578$.

8. Vazquez FL, Guerra LM, Vitor ES, Ambrosano GMB, Mialhe FL, Meneghim MC, et al. Referência e contrarreferência na atenção secundária em Odontologia na cidade de Campinas, SP. Ciência \& Saúde Coletiva 2012. Disponível em URL: http://www.cienciaesaudecoletiva.com.br/artigos/artigo_int.php?id_artigo=11630.

9. Alves VS. Um modelo de educação em saúde para o Programa de Saúde da Família: pela integralidade da atenção e reorientação do modelo assistencial. Interface 2005; 9(16):3952 .

10. Brasil. Conselho Nacional de Secretários de Saúde. Sistema Único de Saúde. Conselho Nacional de Secretários de Saúde, Brasília. CONASS, 2007.

11. Gomes R, Nascimento EF, Araújo FC. Por que os homens buscam menos os serviços de saúde do que as mulheres? Cad Saúde Pública, 2007; 23(3):565-74.

12. Organização Pan-Americana de Saúde (OPAS). Extensão da cobertura dos serviços de saúde mediante o uso das estratégias de assistência primária e participação da comunidade. In: 4⿳亠丷a Reunião Especial de Ministros da Saúde das Américas [online]; 1977 Set 26-27; Washington.

13. Rocha CR, Bercht SB. Estudo do abandono do tratamento odontológico de um serviço público de Porto Alegre: o Centro de Saúde Murialdo. Rev Fac Odontol Porto Alegre 2000; 42(2):25-31.

\section{Endereço para correspondência}

Fabiana de Lima Vazquez Faculdade de Odontologia de Piracicaba, FOP/ UNICAMP

Avenida Limeira, 901, Areião

13414-903 Piracicaba, SP

Fone: (19) 2106-5278

E-mail: fabilivazquez@gmail.com

Recebido: 02/09/2013. Aceito: 22/10/2013. 